\title{
SIMULACIÓN DE LOS PROCESOS CONTROLADORES DE LA REPRESA SALVAJINA
}

\author{
Heidy Rueda* \\ Irene Tischer**
}

* Ingeniera de Sistemas

**Ph.D. Profesora Asociada - Escuela de Ingeniería de Sistemas y Computación - Universidad del valle

\section{RESUMEN}

El objetivo del presente trabajo es mostrar, como se puede aplicar las metodologías de la Dinámica de Sistemas para el mejoramiento de un sistema real. El estudio se centra en el funcionamiento de la represa Salvajina. Construimos un modelo que representa el sistema real. Experimentando con el modelo llegamos a proponer una estrategia de manejo de la represa que permite satisfacer la demanda de agua potable en Cali en épocas de sequía prolongada. 


\section{ABSTRACT}

The objective of the present study is to show how methods of systems dynamics could be applied to improve a real system. The study is centered to the operation of the Salvajina dam. We construct a model that represents the real system. As we e experimented with the model we get to propose a strategy about how to operate the reservoir in such a way that the water demand of the city of Cali is guarantied even in long dry periods.

\section{INTRODUCCIÓN}

La represa Salvajina, que almacena las aguas del río Cauca, está situada en el corregimiento de Suárez, municipio de Buenos Aires en el Departamento del Cauca. El embalse tiene una capacidad total de $908.6 \mathrm{Mm}^{3}$; su volumen útil es de $753 \mathrm{Mm}^{3}$.

El proyecto de regulación del río Cauca en el momento de su planeación tuvo como objetivo principal evitar las inundaciones provocadas por los desbordamientos del mismo. El beneficio esperado era no sufrir las pérdidas causadas por las inundaciones y aumentar el rendimiento en la productividad agropecuaria a causa del mejoramiento en la calidad de los suelos, por lograr un drenaje apropiado y detener el avance de la salinidad. La superficie de tierra así beneficiada consta de una extensión de 131.700 hectáreas compuestas por 68.900 hectáreas que se inundaban directamente y 62.800 hectáreas más que sufrían limitaciones en su drenaje. Para regular las crecidas de abril - mayo y octubre - noviembre, la represa se evacúa actualmente en dos períodos del año.

Otro objetivo importante es la generación de energía eléctrica. La potencia instalada es de 270.000 KW en tres unidades de $90.000 \mathrm{KW}$ cada una. La generación media es de 1.050 millones de KWh por año. En los últimos años, otro objetivo está ganando siempre más importancia: con el crecimiento continuo de la ciudad de Cali, han aumentado también los requerimientos de agua, de los cuales el río Cauca es una de las fuentes más importantes. En la represa se puede ahora almacenar agua para su uso en tiempos de sequía. La eficiencia del suministro de agua se mide en términos del caudal promedio en Juanchito, Cali, que tiene que superar mensualmente un valor mínimo, equivalente a $130 \mathrm{~m}^{3} / \mathrm{seg}$, definido para garantizar el cumplimiento de la demanda de agua en la ciudad.

La represa de Salvajina es manejada por la Corporación Autónoma Regional del Valle del Cauca (CVC) y por la Electrificadora del Pacífico S.A. (EPSA), quienes son las encargadas de tomar las decisiones concernientes a todas las actividades que allí se llevan a cabo. Es así como cada mes se reúnen dos personas representantes de cada una de las partes. La CVC vela por el control de inundaciones en las zonas aledañas a la represa, mientras que EPSA se preocupa por la generación de energía para la venta.

Cada una de las partes teniendo muy en claro sus objetivos se reúnen para analizar lo ocurrido durante el mes anterior, verificar las necesidades inmediatas y fijar metas a cumplir para finales del mes; a partir del momento en que se fijan los objetivos, día a día se activan las compuertas, para que según la entrada, la salida sea adecuada para poder cumplir con lo establecido.

El presente estudio pretende representar como modelo, el sistema que se conforma alrededor de la represa de Salvajina y usar este modelo para plantear y evaluar diversas estrategias de control y analizar su factibilidad, con el fin de encontrar una propuesta clara y concisa de las medidas que se podrían tomar para evitar los problemas que actualmente se vienen dando en ciertas épocas de graves sequías o niveles altos de pluviosidad. 
La metodología empleada es la dinámica de sistemas que permite, a partir de una concepción integral del sistema construir un modelo en forma de un sistema dinámico. El modelo se valida para asegurar que describe correctamente la realidad en los aspectos relevantes para el problema. Aprobada la fidelidad del modelo, se puede usar para ensayar y evaluar estrategias para posibles situaciones críticas.

\section{EL MODELO}

El modelo que se desarrolló consiste de dos componentes: el sistema hidráulico y el sistema administrativo. En el sistema hidráulico consideramos los flujos y el almacenamiento de agua: el caudal del río Cauca al entrar y salir de la represa, los caudales de los ríos tributarios debajo del embalse, la precipitación que cae en la superficie de la represa y la evaporación por la cual se disminuye la cantidad de agua almacenada. Aquí también son de considerar las características de la represa misma: su contenido actual y mínimo y máximo de agua que se puede almacenar. La dinámica de este subsistema está dada por los caudales que a su vez dependen del clima y del control de la salida de agua de la represa.

El sistema administrativo está presente en el modelo por el control que ejerce sobre la salida de la represa. Actualmente, existen estrategias claramente definidas sobre la cantidad de agua que se deja salir del embalse mensualmente. En los diferentes escenarios evaluaremos $y$ modificaremos precisamente estas estrategias, con el fin de hacer propuestas que permitan alcanzar los objetivos del sistema también en tiempos de condiciones climáticas extremas.

La figura 1 ilustra el modelo usado. Muestra que el agua represada está determinada por los caudales de entrada y salida (y por supuesto, del valor inicial), y que está afectada por la precipitación que cae en la superficie y la evaporación. Si al caudal de salida le sumamos la contribución neta de los tributarios, obtenemos el caudal del río Cauca en Cali (Juanchito), la variable que mide la bondad del sistema en cuanto al suministro de agua para Cali. La administración de la represa interfiere ejerciendo control sobre el caudal de salida. La estrategia que define el caudal de salida en un momento determinado, depende del tiempo (teniendo en cuenta la pluviosidad típica de los diferentes meses), del volumen de agua que la represa contiene actualmente y del caudal promedio del río Cauca en el mes anterior.

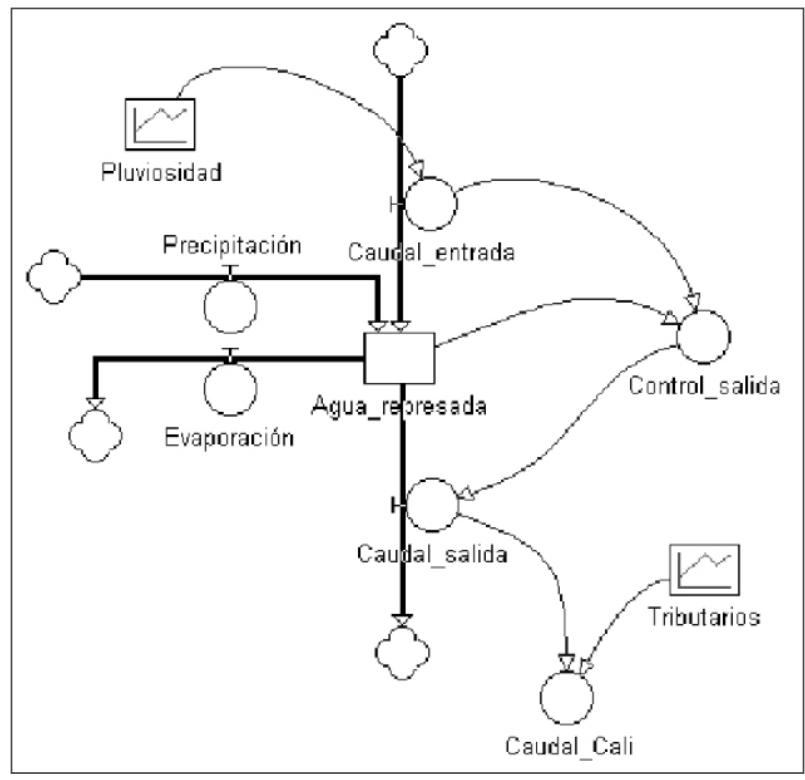

Figura 1. El diagrama de Forrester para el sistema Salvajina. Se usa el símil hidrodinámico para presentar sistemas dinámicos. Los elementos usados son niveles (representados por rectángulos), flujos (usan el símbolo de una válvula) y variables (los círculos ) y tablas. Las flecha indican las relaciones funcionales entre estos

El sistema correspondiente de ecuaciones se puede representar en forma simplificada así:

\footnotetext{
d Agua_represada $/ \mathrm{dt}=$ Caudal_entrada $(\mathrm{t})$-Caudal_salida $(\mathrm{t})+$ + Precipitación (t) -Evaporación (t) 
El modelo se ha implementado en el programa Evolución 2.0, el cual fue diseñado especialmente para simular sistemas dinámicos, permitiendo el cambio de valores o componentes para lograr su validación y así pasar al cambio de escenarios en donde el mismo modelo es sometido a distintas condiciones tanto normales como extremas, para luego analizar su comportamiento y llegar así a conclusiones objetivas.

Este modelo ejecutado con información reciente, es una representación del sistema en su funcionamiento actual. La bondad de esta representación se evalúa en un proceso de validación, que compara el desempeño del sistema en el pasado con el del modelo, ejecutado con la misma información histórica. La figura 2 muestra el volumen real de agua en la represa y el volumen simulado durante los años 1986 hasta 1995. Realizando el análisis estadístico para el error entre estas dos variables se verifica que cumple con las exigencias que generalmente se pide de un error estadístico (independencia, normalidad y media cero). El valor de la desviación es relativamente alto (134 $\mathrm{Mm}^{3}$ en comparación con el volumen máximo de la represa alrededor de $906 \mathrm{Mm}^{3}$, lo que significa un $18 \%$ ) se puede posiblemente explicar con la discrepancia entre la política de control establecida mes a mes y la práctica diaria del manejo de la salida, para la cual no se conocen con exactitud los datos.



Figura 2. Comparación entre las variables volumen de agua real y simulado en los años 1986 - 1995

\footnotetext{
${ }^{1}$ Evolución 2.0, desarrollado por el grupo SIMOM de la Universidad Industrial de Santander - UIS
}

\section{DEFINICIÓN Y EVALUACIÓN DE LOS ESCENARIOS}

El presente trabajo se centra en el estudio del sistema Salvajina en tiempos de sequía prolongada. Muestra hipótesis es que en estos tiempos, el suministro de agua para Cali está en peligro. Esta hipótesis se justifica por un análisis del caudal medido en Juanchito, Cali, entre los años 1986 1995. Aunque no se trata de un período de sequía extraordinaria, el límite de 130 $\mathrm{m}^{3} / \mathrm{seg}$. que garantiza el suministro adecuado de agua, se pasa por debajo varias veces (ver figura 3).

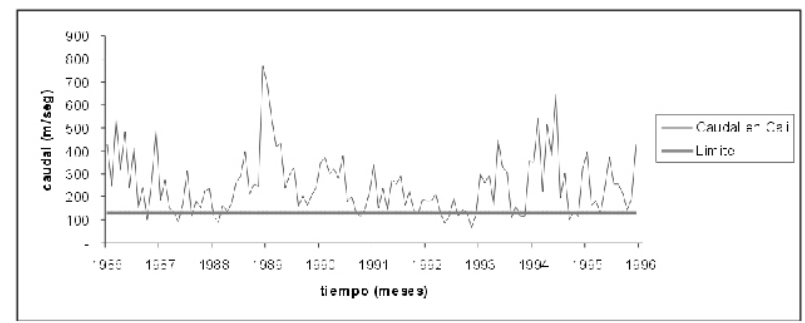

Figura 3: Comportamiento del caudal promedio en Juanchito, Cali. Valores por debajo del caudal mínimo significan que el suministro de agua no es adecuado.

\section{Escenario 1: El sistema en sequía prolongada, manteniendo la estrategia actual de control de salida}

Para este caso definimos un escenario de sequía prolongada, es decir, simulamos el sistema durante cuatro años muy secos. La información de entrada que se debe modificar para efecto de la simulación de este escenario son los caudales del río Cauca y de los tributarios, que es donde más se ve reflejada la influencia del clima. Una modificación de estos afecta directamente tanto el nivel de la represa como el flujo medido en Juanchito.

Para garantizar que las condiciones de extrema sequía, generadas artificialmente para este escenario, no se alejen demasiado de la realidad, se procedió de la siguiente manera: de las series de datos mensuales históricos se seleccionó los cuatro valores más bajos en cada mes, ordenando estos cuatro datos aleatoriamente. El primer dato de cada mes corresponde entonces 
al primer año simulado, el segundo dato al año 2 etc., generando así la información de los cuatro años de sequía.

En este escenario mantuvimos la estrategia para el control de la salida de la represa y observamos la influencia del caudal del Cauca en Cali en los cuatro años secos (ver figura 4).

Puede verse claramente cómo el control que hasta el momento se viene realizando no resulta muy preventivo. Al momento de presentarse un comportamiento imprevisto de los caudales, la estrategia actual busca conservar un nivel aceptable en la represa, a costo de lo que ocurre en la estación de Juanchito. En sólo unos cuantos meses de sequía, llegarían momentos en que la salida se ha disminuido al máximo, causando la disminución del caudal en Juanchito por debajo del límite, y como consecuencia se ocasionaría escasez de agua, energía y un alto grado de contaminación en las zonas que dependen de la represa.

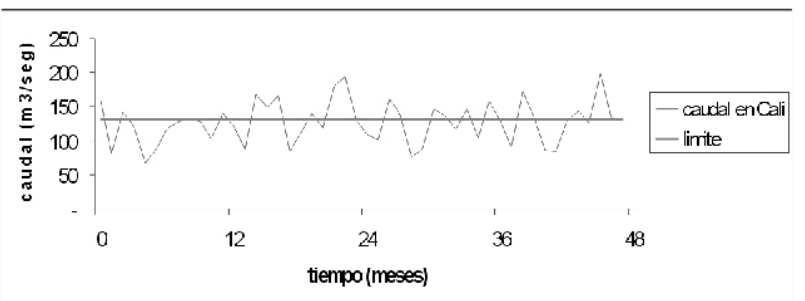

Figura 4: Comportamiento simulado del caudal promedio en Juanchito durante 4 años secos. Constantemente está faltando agua para Cali

\section{Escenario 2: El sistema en sequía prolongada, modificando la estrategia actual de control de salida}

En este escenario queremos estudiar alternativas que tienen el objetivo primordial de atender la solicitud de agua, para lo cual tienen que respetar ante todo el límite impuesto. Una primera idea era la de usar la misma estrategia actual para determinar el caudal de salida, pero aumentándolo si fuese necesario para respetar siempre el caudal mínimo establecido para Cali. Una simulación de esta estrategia para tiempos de sequía muestra que no es factible: Cumpliendo con la demanda de Cali, se desocupa la represa durante los primeros ocho meses de la sequía.

Por eso, una alternativa viable debe ser balanceada: Por un lado debe tratar de cumplir con el límite para evitar la escasez de agua en las zonas que siguen a la represa y que dependen del caudal que de allí provenga. A la vez se debe conservar un nivel de la represa que aunque esté por debajo de lo presupuestado, sea aceptable en términos de funcionamiento de la misma.

La propuesta que se presenta aquí se aplica en los momentos en que bajan las condiciones normales de control, situaciones en las cuales no podría satisfacer el límite mínimo establecido y medido en Juanchito. Se propone implementar una salida extra que se active en los meses en que el caudal en Juanchito baje a menos de 135 $\mathrm{m}^{3} / \mathrm{seg}$ y sólo si el volumen de la represa, después de aplicar esta medida, continúa siendo mayor a $153 \mathrm{Mm}^{3}$ (por debajo de este valor el embalse se considera muerto, ya no es operable).

La cantidad que se define como salida extra debería concertar entre los requerimientos de agua en Cali y las existencias de agua en la represa. Por tal razón la definición de la estrategia es un poco complicada (ver figura 5). La idea es la siguiente: Si la salida normal debe ser cero para alcanzar el volumen propuesto traería como consecuencia que en el siguiente mes tampoco se alcanzara el valor del caudal en Juanchito, por lo que en este caso se propone dejar salir una cantidad moderada que aunque es menor que el equivalente a $135 \mathrm{~m}^{3} / \mathrm{seg}$., será suficiente para tratar de estabilizar este caudal en el siguiente mes sin que el nivel de la represa baje del mínimo establecido.

Si la salida no es cero y hay suficiente agua en la represa se puede permitir que salga el doble de lo estipulado, con el fin de normalizar la situación lo más rápido posible, pero si no hay suficiente agua para esto, al menos se debe permitir que salga un porcentaje extra para aliviar en algo el panorama actual. 


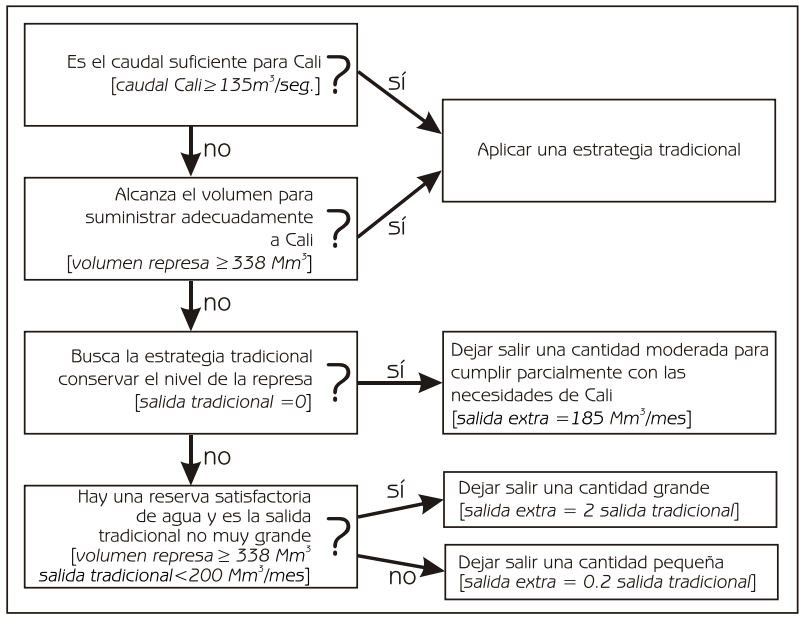

Figura 5: Árbol de decisión, que describe la estrategia propuesta para situaciones de escasez de agua en Cali.

La siguiente figura contrasta el comportamiento del sistema bajo la estrategia modificada con el del sistema tradicional en la simulación de la sequía prolongada. Son evidentes los beneficios de esta nueva forma de control, ya que la situación de escasez de agua para Cali es mucho menos frecuente.



Figura 6: Comparación de los caudales medios en Cali empleando el método tradicional y la nueva propuesta.

La ventaja de esta solución es que se activa la salida extra sólo cuando el caudal en Cali resulta por debajo del límite. Esto hace que en tiempos normales la represa siga funcionando correctamente, ya que los parámetros de control nose modifican.

Siguiendo esta nueva forma de control se tendrá como resultado una atención rápida al momento de no poder alcanzar el límite que probablemente en tiempos de prolongada sequía no se podrá respetar totalmente, pero el panorama sería más alentador y en todo caso se conservaría algo para poder responder en los siguientes meses hasta que el clima se vuelva a normalizar.

\section{CONCLUSIONES}

Para garantizar el adecuado funcionamiento de la represa es indispensable que la práctica se ciña estrictamente a una política clara y bien difundida, dentro de la cual sería adecuado tener un plan específico para afrontar las situaciones críticas. Por el modelo de simulación se pudo comprobar que la estrategia actual definida por la administración de la represa daría resultados satisfactorios siempre y cuando se siguiera al pie de la letra, al menos en situaciones climáticas no extremas. En tiempos de sequía está en peligro el suministro adecuado de agua para Cali, por esta razón se evaluó en esta investigación una política alterna que solamente se hace efectiva en tiempos de sequía prolongada dando prioridad al cubrimiento de la demanda de agua por encima de la demanda de generación de energía, para establecer un equilibrio entre los servicios prestados.

Esta política se evaluó mediante el modelo de simulación, sin embargo antes de implementarla sería recomendable tener en cuenta:

Mo basar la estrategia de control solamente en lo ocurrido en el mes inmediatamente anterior, como se ha venido haciendo. Involucrando en la estrategia de control mensual más información del pasado podríamos tener un modelo más confiable para la predicción de los caudales.

Se propone ensayar en este contexto el poder predictivo de los modelos de series de tiempo o redes neuronales con respecto al comportamiento de los factores climáticos.

Los resultados obtenidos con la simulación que se hizo mes a mes (por ser ésta la forma en que 
actualmente se maneja la información), podrían mejorarse si se hiciera día a día. Esto permitiría una mejor aproximación a las condiciones reales, con lo cual sería posible establecer la política de manejo diario que siga el lineamiento de la estrategia mensual para ver en una forma más clara dónde podría estar fallando y así tener un mejor control sobre las situaciones críticas.

El caso presentado aquí es un ejemplo de cómo se pueden aplicar las metodológicas de la Dinámica de Sistemas para resolver problemas reales relacionadas con la evaluación de estrategias. La herramienta de simulación permite realizar experimentos con el modelo computacional en situaciones donde es imposible o no deseable por limitaciones de tiempo y dinero, sujetar el sistema real a pruebas. Se espera este trabajo anime a otras personas a realizar estudios en el campo de la Dinámica de Sistemas, pues presenta amplias posibilidades y tiene gran aplicación en muchas áreas de nuestro medio.

\section{BIBLIOGRAFÍA}

Avances en Recursos Hidráulicos Mo. 3, agosto 1995. Programa de Postgrado en Aprovechamiento de Recursos Hidráulicos. Universidad Macional de Colombia. Medellín.

DYMER R., Isaac. Dinámica de Sistemas y Simulación Continua en el Proceso de Planificación. 1a. Edición, enero 1993.

EPSA, Empresa de Energía del Pacífico S.A. E.S.P. Mormas de Operación de la Central de Salvajina. Abril, 1977

LATORRE E., Emilio; "Enfoque de Sistemas Conceptos y Aplicaciones". Universidad del Valle, 1993.

LATORRE E., Emilio; "Teoría General de Sistemas"; Editorial Universidad del Valle, 1996.

LEVIM, Richard I.; RUBIM, David A. Estadística para Administradores, Sexta Edición; Prentice Hall, 1996. p. 852-942.
MARTíneZ, S.; REQUEMA, A. "Dinámica de Sistemas". De. Alianza, Madrid, 1986.

FOGATA Katsuhiko. Dinámica de Sistemas. Prentice-Hall Hispanoamericana, S.A. México, Englewood Cliffs.

PIDD, M.; "Computer Simulation in Management Science". John Wiley, Mew York, 1992.

RUEDA G. Heidy M.; "Simulación de los Procesos Controladores de la Represa de Salvajina"; Tesis de Grado en Ingeniería de Sistemas, Universidad del Valle, Cali, 1998.

SALVAJIMA "El Parto de una Quimera". Corporación Autónoma Regional del Cauca, Santiago de Cali, noviembre de 1985.

SHAMMOM, Robert E. Systems Simulation, the Art and Science. Prentice-Hall, Inc. Englewood Cliffs, New Jersey, 1975. 